\title{
Review of: "Barley guanine nucleotide exchange factor HvGEF14 is an activator of the susceptibility factor HvRACB and supports host cell entry by Blumeria graminis f.sp. hordei"
}

\author{
Stefan Kusch ${ }^{1}$, Björn Sabelleck ${ }^{1}$ \\ 1 Rheinisch Westfälische Technische Hochschule Aachen
}

Potential competing interests: The author(s) declared that no potential competing interests exist.

This manuscript describes HvGEF14 as an activator for HvRACB, a ROP involved in polar cell rearrangement in the powdery mildew interaction. By describing HvGEF14 as guanine nucleotide exchange factor of RACB, this study adds further knowledge about the molecular basis of powdery mildew infection in barley.

- The phylogenetic analysis is rather limited (based on only three species, one dicot and two monocots), while the authors make rather broad conclusions about the phylogeny of PRONE-GEFs in plants based on their findings, and attribute their improved phylogeny to the phylogenetic method and curated genes. The authors should either enrich their analysis with PRONE-GEFs from at least another dicot and a nonangiosperm plant, or be more critical about their conclusions in this case by highlighting the limitations of their dataset.

- The authors describe that HvGEF14 is mostly expressed in the epidermis and induced during B. graminis infection, which is why they continued with this GEF. However, what is missing is expression analysis for all other GEFs to support this. Since I could not find Table S6 in the supplements (they only contain supplementary figures), I cannot judge if HvGEF14 is really the best candidate based on expression pattern. In any way, other GEFs should be tested by qRT-PCRs to demonstrate that they do not accumulate in epidermis and/or are non-responsive to Blumeria infection.

- Lines 481-483: I am not convinced that using N. benthamiana automatically reduces background from endogenous components. To support the claim, the authors should detect all PRONE-GEFs and other GTP signaling components in N. benthamiana (whose genome and proteome are available) and demonstrate that they are significantly different.

- I noticed a number of typographic and formatting errors, suggesting incomplete editing of the manuscript. E.g., in lines 224 and 384, the font type suddenly changes. Also, numerous typos (e.g., line 41 should read "led to interaction", line 218 Qiagen should be written without u, line 209+333 inkScape is written Inkscape, line 204 date is written in German instead of English form, in many instances a space is missing between number and unit, like $5 \mathrm{ml}$ should be $5 \mathrm{ml}$, line 298 leave should read leaf). 
Line 202, ML analysis can be mistaken for machine learning - do not abbreviate in the subheading and preferably avoid the abbreviation altogether.

- Line 542-543: "AtGEF14 expression has also been reported" - this statement contains no information, and no information about this is provided in the following sentences.

- A general suggestion is the revision of the nomenclature since it is not consistent. For example, HvGEF 124-485 is written in different variations; Fig. S3: HvPRONE14 (124-485), Fig. S4: PRONE 124-485, and Fig. 4: HvGEF14 124-485.

- Fig. 1: The number of bootstrap replications should be indicated in the legend. Also, the branch length calculations are not explained and a size bar/legend is missing.

- Fig. 2: The legend promises phosphorylation sites (green), but I cannot find a single green site in the alignment. The alignment is lacking a suitable Arabidopsis GEF in comparison. Also, why are these domains indicated in Fig. 2 not also indicated in the alignment in Fig. S2?

- Fig. 3: qRT-PCR for another GEF as control is missing.

- Fig. 4: The dataset in Fig. 4 is incomplete since only the combination RACB (bait) against the eV control (prey) is shown. The combination eV (bait) vs GEF14/GEF14 PRONE should be shown to complete this dataset. This control is present in Fig. S4 and, therefore, not much work to implement. The figure size can be maintained if only one dilution on - L-W-H is depicted.

- The Fig. 4 legend stated that the original image is found in Fig. S1 (instead of Fig. S4), needs to be corrected to avoid confusion.

- As a helpful suggestion, the -L-W control plate not only shows a successful transformation. It is also necessary to show that the expression of a bait or prey protein does not cause any yeast growth defects. This could be one reason why some combinations look weaker since the growth on -L-W is also reduced. But this observation does not change the statement that interaction is present.

- Figures 5, 6, and 7: EV is a negative control, but not sufficient. These experiments should be done with another protein (preferably a related but non-interacting GEF) to demonstrate specificity of the observed interaction and relocalization effects.

- Fig. 7: the RACB DN construct should be added as a control in this experiment.

- Fig. 8: Another GEF should be used as negative (no effect) control. Also, a proper positive control (resistant/susceptible control) is lacking and required to show that effects are not an over-expression artifact.

- Fig. S4: It would be helpful to state why you use the combination RIC171 vs RACB CA. I guess it is a positive control.

- Fig. S5: First of all, a loading control (like in Fig. S7, Ponceau staining) is missing. Also, an explanation for what F, P and E are indicating would be helpful for the reader. In part A, KDa is written normally, whereas in part B is written in bold. It would be beneficial to indicate which $\mathrm{Y} 2 \mathrm{H}$ experiment corresponds to which blot. In part A, an indication for the bait is missing in the last two lanes. I guess it is the eV control? 
- Fig. S6: The abbreviation Ade is inconsistent with the rest. The change to a simple A would make it more consistent and is usually used for Ade. The control eV vector (bait) vs GEF14 is missing, but it was shown before that no interaction takes place. Because of that not necessary.

- Fig. S7: The upper part of part A is cut off in the bioRxiv manuscript. I guess this blot belongs to the $\mathrm{Y} 2 \mathrm{H}$ in Fig. S6, but why are there HvRIC171 and RACB CA indicated? Shouldn't they be in Fig. S5? 